Journal of Social Sciences 4 (3): 189-195, 2008

ISSN 1549-3652

(C) 2008 Science Publications

\title{
Electronic Monitoring: The Employees Point of view
}

\author{
${ }^{1}$ Hamed Al-Rjoub, ${ }^{2}$ Arwa Zabian and ${ }^{1}$ Sami Qawasmeh \\ ${ }^{1}$ Irbid National University, Irbid, Jordan \\ ${ }^{2}$ Jadra University, Irbid, Jordan
}

\begin{abstract}
Electronic monitoring tools provide managers with the ability to continuously evaluate and measure their employee's performance. The employee's productivity will be increased and his work will be more accurate. This study has examined the effects of electronic monitoring on the employee's point of view. We have assume that the employee will be more productive when he is electronically monitored. On the other hand, the manager will be able to control the employee's performance efficiently and will be more satisfied by the employee's works. We have conducted a survey that led us to believe that employee's work will be improved when he is electronically monitored. Our results suggest that electronic monitoring has been accepted by many employees as a technology tools that help in improving the employee's behavior.
\end{abstract}

Key words: E-monitoring, performance management, monitoring tools, productivity

\section{INTRODUCTION}

The Internet has given a powerful window on the world to every office worker with a personal computer and a web connection, it is also a powerful distraction. The very definition of what is private is up for grabs as lines between working hours, personal time and home life. The informality of the medium lends itself to the rapid transfer of a company's trade secrets outside the organization. What they can't send via E-mail angry employees can be carried on a floppy disk.

The managers are much more aware and much more nervous about productivity. For that, they provide E-mail services to their employees as an efficient means of facilitating both intra company communication and communication with the outside client base. E-mail serves to increase the efficiency of today's work place because it is inexpensive to provide, simple to install and easy to use. However, despite these efficiencies, this technological advancement is also creating collateral problems concerning issues of employee privacy that today's legal environment appears unprepared to solve.

In fact, many employees operate under the false assumption that personal E-mail messages sent from work are protected from their employer's scrutiny.

All today's businesses are constantly attempting to increase employee efficiency, for that the managers are becoming more concerned with improving their employees' hourly productivity for that he provides for him the most current technology to improve their performance that consecutively increases the organization performance. This role of the manager is known as performance management.

Armstrong and Baron $^{[1]}$, define performance management as' a process which contributes to the effective management of individuals and teams in order to achieve high levels of organizational performance.

Performance management is a systematic process which an organization involves its employees, as individuals and members of a group, in improving organizational effectiveness in the accomplishment of the organization mission and goals. Employee performance management includes:

- Planning: Means work and setting expectations continually for groups and individuals to channel their efforts toward achieving organizational objectives

- Monitoring: Will mean consistently measuring performance and providing ongoing feedback to employees and workgroups on their progress toward reaching their goals

- Developing: Means increasing the capacity to perform, periodically through training

- Rating: Summarizing employee performance from time to time

- Rewarding: Means recognizing employees, individually and as members of groups, for their performance and acknowledging their contributions to the organization's mission 
Great work has been done to ensure that these regulations would complement and not conflict with the kind of activities and actions practiced in effective organizations.

In this study we will study the role of Emonitoring in increasing and improving the performance of the employees.

The manager often monitor employee telephone calls and some companies also record the time each employee spends on bathroom breaks. These attempts to monitoring employee behavior, are silly as some may appear, represent aspects of legitimate struggle between the employer's ability to conduct its business operations and the employees privacy rights, between worker efficiency and worker sanity and between technological advancement and current laws operating behind the technological curve. In fact, the manager has many legitimate reasons for desiring to monitor their employees' E-mail usage to maintain employee productivity.

Many attempts have been done in the context of Emonitoring that concentrate on the negative consequences of E- monitoring that affected the employees' performance. In which the psychological aspects and the stress and the violation of privacy of the employees that are caused by E-monitoring have been studied. The positive effect of E-monitoring from the employees' point of view which ensures the peacefulness of the work and affects performance of the employees has not been studied. For that we will focus on the idea that E-monitoring is useful from the employees' point of view and is also useful to the organization. We have done an induction of the opinions of the employees by distributing a questionnaire to different categories of employees. Our results reveal the desire of large portion of employees to be monitored for different reasons some of which are the elimination of recommendations and the evaluation of the contributions and many other reasons will be discussed and analyzed in our study.

E-monitoring toward performance management: In 1960 , the concept of electronically monitoring in the United States, Canada and the United Kingdom was introduced to reduce prison population. Later, the use of electronic monitoring is disseminating to many other different fields such as business, home and jurisdiction monitoring....

In general, the manager has the right to be able to check the performance and activities of an employee. Merely this task is accomplished by physical checking of the employee performance and watching the way he or she works or it is done by measuring the amount of work carried out in a given period of time. This practice has evolved with the development of technology and it is now possible to observe the activities of an employee using various electronic methods. The electronic methods which are used now vary according to the task being performed. These include methods that can be used to monitor telephone calls and to monitor the employees with video equipment, scan e-mail and track Internet usage. In addition, there are systems which can keep track of the keystrokes on computer terminals.

E-monitoring is the use of electronic technology to directly monitor, supervise and evaluate employee's performance

E-monitoring technology can be categorized into three different categories:

Category1: Focus on performance, such as measuring the use of computer time, the telephone conversation content or measuring keystrokes.

Category 2: Focus on employee behavior, such as measuring the use of resources, testing an employees predisposition to error, or tracking worker location via identification badges

Category 3: Focus on employee characteristics, such as their truthfulness or the state of their health.

Certainly, the manager has the right to monitor employees' work for performance evaluation purpose. However, do they have the right? Is it ethical? Is it good for the employees-manager relation, or does it even make economic sense to use computer to monitor employees constantly and to collect detailed statistics? Another important question can be asked here, should the employee know that she or he is monitored? Is it a privacy violation of the manager to monitor their employees without their knowledge?

Motivation of E-monitoring: Today, almost all jobs have the potential to be subjected to electronic surveillance. Some jobs are particularly susceptible to monitoring practices more than others. These can range from the office worker whose supervisor reads his or her E-mail message to the grocery store cashier whose bar code scanner records the speed at which he or she is working.

There have been several reasons suggested for the predominance of surveillance. One suggestion is the simple fact that technological possibilities exist, other reasons could be performance evaluation, or cost control of the use of company resources or protection of business information or security and safety. 
In particular, the organization might monitor the employee e-mails for the following reasons:

- To ensure that electronic communication facilities provided by the company solely for company business

- To be able to define who may review the company information, the purpose for which the information may be used and that the information may be stored on a separate computer

- To avoid the sending of any discriminatory, offensive, or unprofessional message content

- To ensure that the accessing of any Internet site that contains offensive or discriminatory content is unused

- To avoid the posting of personal opinions on the Internet using the company's access

These reasons are considered minimum standard that motivate monitoring of e-mail and Internet usage of the employees.

However, productivity concerns are also at stake in this issue. It is well understood that employees who are not supervised by some means or other can waste a considerable amount of time. Wherever, performance management reminds us that being busy is not the same as producing results.

Consequences of employees monitoring: Emonitoring has positive and negative effects from employee's point of view as well as the company's point of view.

- From the employees' point of view the most devastating effect of employees' monitoring is the fear of losing their jobs. In addition to ethical and social issues ${ }^{[2]}$ and many other negative consequences some of them are:

- Lowest interest in the job that transforms the work place to become mistrust and hostile work environment. That makes the employees feel less satisfied with their job and start looking for other secure job opportunities

- Absenteeism that means the number of people taking off of work for personal reasons is on the rise

- Privacy invasion: employees of many organizations are exposed to many types of privacy invasive monitoring while earning a living. However, these activities may diminish employment moral and dignity and increase stress and may lead to lawsuit cases
- Emphasis on speed and quantity instead of quality

- Workers who are subjected to computer monitoring constantly feel pressure to perform and thus are more likely to succumb to stress related illness

- Worker productivity may actually decrease to meet the goal of the measurement being made, worker may cut off customers on the phone line, enter incomplete job

- Lack of trust between employees themselves, between employees and supervisors and between supervisors and managers

- Alienation: sociologists define the concept of employee alienation as a lack of worker freedom and control, purpose and function and self involvement in their job ${ }^{[3]}$

- Increased stress can often lead to physical symptoms. In a study by the department of Industrial Engineering, University of WisconsinMadison, higher levels of stress in monitored employees resulted in an increase in somatic complaints, including a $27 \%$ increases in occurrences of pain or stiffness in shoulders, a $23 \%$ increases in occurrences of neck pressure and $21 \%$ increases in back pain experienced by employees. Such stress and stress related symptoms can create medical expenses, lost time and absenteeism ${ }^{[4]}$

In addition to general negative effects listed above, the knowledge that employees are being watched, listened or otherwise monitored can create a negative workplace atmosphere undermining employee moral and creating division between employees and management. Despite management views of electronic monitoring as a means to improve consistency in employee performance evaluation, employer frequently question the fairness of their employees' use of electronic monitoring to review their performance.

A perceived lack of procedural fairness in monitoring can lead to undesirable negative employee reactions such as withdrawal, sabotage, resignation, or some other format diminished organizational citizenship.

On the other hand, an electronic monitoring system is more likely to find acceptance with employees if the monitoring practices are regarded as relevant to their work. Those whose favor computer monitoring justifies it with several reasons. Among them are:

- The computer is not biased and has no favorites. Thus, the performance evaluation is objective

- Computer monitoring often provides instant feed back on performance, allowing employees to 
quickly adjust their work habits and improve productivity

- monitoring may provide substantial cost savings. Individuals whose performance is not up to par can be replaced or transferred to other tasks

From this point of view, that is looking to emonitoring from the positive effects and consequences we have conducted, a research by distributing a questionnaire to the employees in different environments. All were without electronic monitoring. Our goal is to take an idea about their opinions in the benefits of e-monitoring and then we have classified our results in three categories: the benefits of emonitoring for the employees them selves, the benefits of e-monitoring for the organization from the point of view of productivity and evolution and the effect of the e-monitoring on the relation between manager and employee. All our results will be analyzed and discussed in the next section.

\section{RESULTS}

To study the social and psychological effects of Emonitoring from the employees' point of view, we distributed a questionnaire in different work environments (private and public universities and banks in Jordan). All visited environments were without electronic monitoring. The questionnaire contains 58 questions with different possible answers. The answers were classified: very low probability, low probability, medium, high and very high probability. For analysis purposes, each probability is assigned a value from 1 to 5 respectively to be able to compute the average and the standard deviation of the results obtained in each answer. The average is calculated as follows:

$$
\begin{gathered}
\sigma={ }_{\mathrm{i}=1} \Sigma^{\mathrm{i}=5} \text { the value } \mathrm{X} \# \text { of frequency / total \# of } \\
\text { frequencies }
\end{gathered}
$$

Then, the results are classified in three main categories. The categories are: favorite, impartial and non favorite. The favorite category represents all the answers with high and very high probability. Low and very low probability are considered as non favorite and the medium answers are considered as impartial. This classification is necessary to extract the opinions of the employee about the E-monitoring as technology that improves his or her behavior, performance and influences the relation between managers and employees or employees themselves.

The benefits of E-monitoring in the employee's behavior: The results shown in Table 1, shows that the employees believe in the e-monitoring as a new technology that can help them to control their behavior (angry....) or to adjust the incorrect behavior during their work with high probability. In addition, 52.9\% of employees agree that E-monitoring ensure the job security with high probability, while $11.8 \%$ only believe the opposite. Table 1, show the employees opinion about the effects of e-monitoring in:

- Control behavior

- Adjusting incorrect behavior and their work habits

- Self control

- Increasing the responsibility

- Increase trust of oneself

- $\quad$ Ensuring job security

- Givining satisfaction and self realization

The results shown in Table 1 are numbered respectively according to the previous points.

From the Table 1 it can be seen that E-monitoring helps the loyal employee to have more satisfaction and security in himself which influences his behavior positively toward his work environment that turns in benefits for the organization.

The effect of E-monitoring in the employee's performance: The results shown in Table 2, show that the agreement with the opinion that E-monitoring increases the employee performance and productivity were not higher than those opposite and that is justified that in general the employees are disinterested in the productivity of the organization. And that confirms our assumption, about the disadvantage of the E-monitoring from the point of low interest in the job and that Emonitoring does not increase the worker productivity. Table 2 Show the employees opinion about the effect of E-monitoring in:

- Increasing productivity

- Eliminating the lazy worker

- Increasing creativity

- Eliminating the negativism and disinterest in the work

- Increasing self development

The results show in Table 2, are numbered respectively according to the previous points.

The effects of E-monitoring in the manageremployees relationship and the employeesemployees relationship: From the results shown in 
Table 1: The effect of E-monitoring on the employee behavior

\begin{tabular}{llllll}
\hline No. & Favorite & Impartial & Non favorite & Average & $\begin{array}{l}\text { Standard } \\
\text { deviation }\end{array}$ \\
\hline 1 & $64.7 \%$ & $35.3 \%$ & - & 3.88 & 0.78 \\
2 & $52.9 \%$ & $41.2 \%$ & $5.9 \%$ & 3.76 & 0.97 \\
3 & $58.8 \%$ & $29.4 \%$ & $11.8 \%$ & 3.71 & 0.98 \\
4 & $58.8 \%$ & $23.5 \%$ & $17.7 \%$ & 3.71 & 1.16 \\
5 & $70.6 \%$ & $17.6 \%$ & $11.8 \%$ & 3.65 & 0.99 \\
6 & $52.9 \%$ & $35.3 \%$ & $11.8 \%$ & 3.59 & 0.93 \\
7 & $64.7 \%$ & $17.6 \%$ & $17.7 \%$ & 3.59 & 0.99 \\
\hline
\end{tabular}

Table 2: The effects of e-monitoring on the employees' productivity and evolution

\begin{tabular}{llllll}
\hline No. & Favorite & Impartial & Non favorite & Average & $\begin{array}{l}\text { Statistical } \\
\text { deviation }\end{array}$ \\
\hline 1 & $41.1 \%$ & $47.1 \%$ & $11.8 \%$ & 3.53 & 1.00 \\
2 & $64.7 \%$ & $5.9 \%$ & $29.4 \%$ & 3.50 & 1.31 \\
3 & $41.1 \%$ & $41.2 \%$ & $17.7 \%$ & 3.41 & 1.17 \\
4 & $47.0 \%$ & $35.3 \%$ & $17.7 \%$ & 3.41 & 1.12 \\
5 & $52.9 \%$ & $23.5 \%$ & $23.6 \%$ & 3.35 & 1.27 \\
\hline
\end{tabular}

Table 3: The effect of E-monitoring in the manager -employees' relationship and employees themselves

\begin{tabular}{llllll}
\hline No. & Favorite & Impartial & $\begin{array}{l}\text { Non } \\
\text { favorite }\end{array}$ & Average & $\begin{array}{l}\text { Standard } \\
\text { deviation }\end{array}$ \\
\hline 1 & $76.5 \%$ & $17.6 \%$ & $5.9 \%$ & 4.06 & 0.69 \\
2 & $76.5 \%$ & $17.6 \%$ & $5.9 \%$ & 3.88 & 0.78 \\
3 & $75.4 \%$ & $18.7 \%$ & $5.9 \%$ & 3.80 & 0.77 \\
4 & $58.8 \%$ & $17.6 \%$ & $23.6 \%$ & 3.65 & 1.16 \\
5 & $58.8 \%$ & $11.8 \%$ & $29.4 \%$ & 3.41 & 1.22 \\
6 & $53.0 \%$ & $23.5 \%$ & $23.5 \%$ & 3.35 & 1.11 \\
7 & $53.0 \%$ & $17.6 \%$ & $29.4 \%$ & 3.29 & 1.16 \\
8 & $47.0 \%$ & $5.9 \%$ & $47.1 \%$ & 3.18 & 1.51 \\
9 & $34.4 \%$ & $41.0 \%$ & $23.6 \%$ & 3.12 & 1.16 \\
10 & $41.2 \%$ & $29.4 \%$ & $29.4 \%$ & 3.12 & 1.05 \\
\hline
\end{tabular}

Table 3, it can be concluded that the E-monitoring improves the state of the employees (discovering the contributors, equality and legality).

However, $47-58 \%$ of people believe that Emonitoring can improve the relationship between employees and only $34 \%$ of people believe that Emonitoring improves the relation between employee and manager and make the work environment more trustful and more friendly that turns in positive effects for the organization. In Table 3 also, it can be noted that $5.9-29.4 \%$ of people believe that E-monitoring has low positive effects for the employees and this percentage is considered as the opposite to E-monitoring for the reasons mentioned.

Table 3 Show the employees opinion about the effect of E-monitoring in:

- Helping in discovering the contributions and performance evaluation will be objective

- Helping in application of laws for all

- Helping in finding a criteria that strength the relation between employees
- Eliminating the influences

- Helping in the acceptance between employees

- Helping in decreasing the differences in opinions

- Eliminating problems between employees

- Increasing the transparency and the clarity between employees and employees manager

- Eliminating the barriers between employees and manager

- Increase the trust between the employees themselves

The results shown in Table 3 are numbered respectively according to the previous points.

Results analysis: The results shown in Table 1-3 are organized in such a way that the average is in ascendant manner.

By analyzing the results shown in Table 1, (column $2,3,4)$ it can be seen that a high percentage of people prefer the use of E-monitoring as a technology that helps in improving the employee behavior. That is justified by the good desire of the employees to impose their opinions in a clear manner and in this way the good employee who works all the time has been expressed in a justified manner. However, analyzing columns 5, 6 and given the chebyshev's inequality ${ }^{[6]}$ in statistics and probability which states that in all random distribution and not just normal one the results must follow the following bounds:

- At least $50 \%$ of the values are within 1.41 standard deviation from the mean

- And at least $75 \%$ of the values are within 2 standard deviation from the mean

Given that, our best results in Table 1-3 that cope with chebyshev's inequality are the results within standard deviation between 0.99-1.16 however the results must be distributed around the means (Fig. 1). So, from Table 1 and Fig. 1, we can see the results of the first question are with standard deviation outside the previous range and it is clear from the high difference between favorite and non favorite people.

Considering that the same people were taken from the same environment and are in the same social level.

From Table 3 and Fig. 2, we can see that the worst results are the results with the standard deviation 1.51 and that is clear because there are similar percentages of favorite and non favorite that represent a large deviation on the mean. This justifies that the sample are the same and the answers must fit in the same range except some particular cases that may not become a phenomena. 


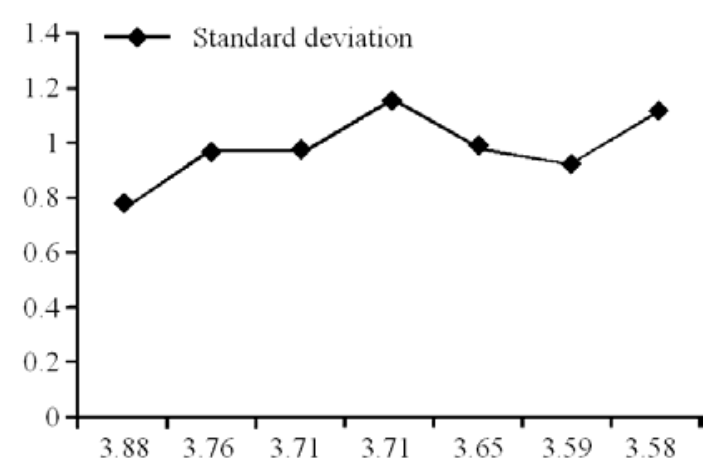

Fig.1: The relation ship between the standard deviation and the mean

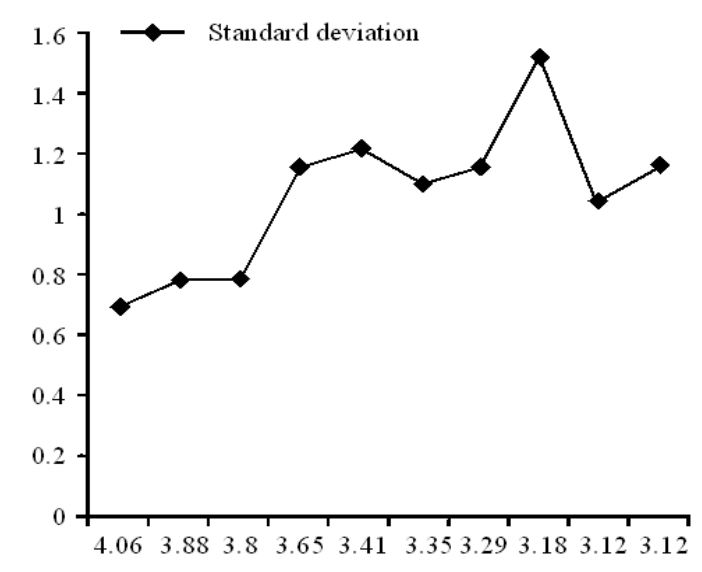

Fig. 2: The relationship of the standard deviation and the means in the relation between employeemanager

Our best results have shown that $58.8-70 \%$ of people are favorite to the E-monitoring and 11.8-17.6\% is impartial and $29.4-11.8 \%$ are non favorite. These values were taken to give the best standard deviation that cope with chebyshev's inequality were $80 \%$ for the first requirement and about $75 \%$ for the second requirement held in our results.

\section{CONCLUSION}

Business has changed in the 21 st century, thanks to the innovations of technology and the Internet. There is no doubt that the Internet is bringing businesses to new customers and provides a wealth research and communication tools. However, the majority of employees at work access are using the Internet for personal reasons. In this study we have analyzed the use of E-monitoring from the employee point of view as a technology that helps in improving his or her behavior during the work and increase his or her productivity. Our results reveal that the employees have a high desire to be monitored to improve their behavior in the work and to improve the relationship between the employees themselves to make the work environment more peaceful and more welcome environment. However, the employees do not care about the organization performance. The results shown in Table 3, show that the percentage of the people that favor E-monitoring is not higher than that for those who do not favor and that is justified as high percentage of people who accept the E-monitoring as a scientific method that improve their behavior and can be useful for the organization but they don't like to be monitored. However, in general the Emonitoring does not increase the productivity of the organization and that is according to the results shown in Table 2 and that is justified because the employee is not meant about the productivity of the organization and that is the role of the manager to think about increasing the organization productivity.

Statistics shows that $64 \%$ of employees admit to surfing the web at work for personal reasons, $97 \%$ of the employees surveyed believe that surfing the net for personal reasons has no effect on their productivity ${ }^{[5]}$. About $65 \%$ of human resource department surveyed had disciplined employees for improper Internet usage. Canadian employees are spending an average of about two hours a week surveying the Internet for personal reasons at work [Angus Reid Group]. Employees surveyed reported using the Internet at work to book a trip (50\%) for education (42\%) research a hobby (41\%), visit a virtual shop or watch a sports event (27\%)[Taylor Nelson......]. One third of workers say they have played computer games during work hours [society of financial Service Professionals].

A study suggests that getting rid of the gossip, jokes can save up to $30 \%$ of the time an employee spends reading the Internet at work [Gartner group].

However, given our results and given the previous statistics the employer is motivated to monitor their employees to be sure that their employees are doing a good job, but employees don't want cooler logged. That's the essential conflict of work place monitoring.

Recent surveys have found that a majority of employers monitor their employees. They are motivated by concern over litigation and the increasing role that electronic evidence plays in law suits and government agency investigations. A 2005 survey by the American Management Association found that three fourths of employers monitor their employees' web site visits in order to prevent inappropriate surfing. And $65 \%$ use software are to block connections to web sites deemed off limits for employees. Over $80 \%$ of employers 
disclose their monitoring practices to employees. And most employers have established policies governing the Internet use [www.amanet.org/research/pdfs/ EMS_summary05.pdf].

Our future research is to study the effect of Emonitoring from the society's point of view and to apply the e-monitoring in the locations which have been studied and to study the differences in the employee's opinions after being monitored.

\section{REFERENCES}

1. Armstrong, M. and A. Baron, 2004. Managing Performance: Performance Management in Action. 2nd Edn., Chartered Institute of Personnel and Development, London. ISBN-10:1843981017.
2. Joseph M. Kizza, 2003. Ethical and Social Issues in the Information Age. 2nd Edn. Springer, New York, pp: 374. ISBN: 0-387-95421-X.

3. Shepard, John. Automation and Alienation. 1971. Cambridge, Ma: MIT Press 1971.

4. Di Tecco, D. et al., 1992. Operator stress and monitoring practices. Applied Ergonomic, 23: 29-34. http://www.ncbi.nlm.nih.gov/pubmed/ 15676846

5. Xylo, INC. Worker Watch-Employee Monitoring cases, Statistics. Survey August 2001. http://www.workerwatch.org/.

6. Grimmett, G. and D. Strizaker, 2001. Probability and Random Processes. Oxford University Press, USA; 3 edition (August 2, 2001). ISBN-10: 0198572220. 